\title{
Workspace Identification of Stewart Platform
}

\author{
S Dasmahapatra, M Ghosh
}

\begin{abstract}
The workspace identification of 6-DOF Stewart Platform has been done in this paper through inverse kinematic modeling. This Stewart Platform has six linear cylinder-piston actuators connected within fixed and the moving platform. The motions of the moving platform such as surge, sway, heave, roll, pitch and yaw have been generated from the combined motions of piston of actuators. The mathematical formulations for Inverse-Kinematic modeling of Stewart Platform have been formulated to find out the individual piston motion for the required platform motion. The platform motions and the actuator motions have been studied for the workspace identification of the Stewart Platform.
\end{abstract}

Keywords: 6-DOF, Inverse-Kinematic, Stewart Platform, Workspace- Identification.

\section{INTRODUCTION}

The Stewart Platform has one fixed platform which is connected with a moveable platform and this connection made by either linear cylinder-piston actuators [1] or some linkage with servo motors [2]. The linear motions of each piston of the actuator make the required motion of the moving platform whereas the rotary motions of each motor with the corresponding linkage transformed to the platform motion. Stewart Platform acts as a parallel manipulator which can generate translation motions as well as rotational motions and their different combined motions. Though the serial manipulator has one of the advantage over the parallel is the larger work space but parallel manipulator has the larger output force, higher rigidity and stiffness [3]. Surge, Sway, Heave- three pure translation motions and Roll, Pitch, Yawthree pure rotational motions along three fundamental axis $\mathrm{x}$, $\mathrm{y}$ and $\mathrm{z}$ assist to develop six degree of freedom motion or 6-DOF Stewart Platform.

The study of Stewart Platform is now a motivating research field because of its modern and precise industrial applications. This manipulator could be used to control the multi-axis vibration of a slender structure [4]. The stabilization problem of a radio-telescope can be resolved by this platform [5-6]. The vibration isolation capability of a

Revised Manuscript Received on February 05, 2020.

* Correspondence Author

Dr. Sibsankar Dasmahapatra, Assistant Professor, Mechanical Engineering Department, Kalyani Govt. Engg. College, Nadia, WB, India.

Mintu Ghosh, Assistant Professor, Mechanical Engineering Department, Bengal College of Engineering and Technology, Durgapur, WB, India.

(C) The Authors. Published by Blue Eyes Intelligence Engineering and Sciences Publication (BEIESP). This is an open access article under the CC BY-NC-ND license (http://creativecommons.org/licenses/by-nc-nd/4.0/)
Stewart platform has been studied by Preumont et al. (2007) [7]. This platform can be used as flight simulator [8], fire detection, and rescue operations [9]. The medical surgery can be performed precisely with this platform [10]. The present study in this paper deals with the inverse kinematic modeling of the Stewart Platform to find out the workspace. This platform has six linear cylinder-piston actuators connected within fixed and the moving platform. The motion of moving platform has been called here as the pose of platform. The platform and its pose have been discussed in the next section followed by the mathematical modeling of the inverse kinematic in the Section III. The inverse kinematic modeling has been formulated to find the individual piston motion for the corresponding pose of the moving platform. In the Section IV, the workspace of the Stewart Platform has been identified and depicted for different types of platform motions.

\section{PLATFORM AND ITS POSE}

The Stewart Platform represented in the Fig. 1 has a fixed platform $\mathrm{F}$ and a moving platform $\mathrm{M}$ on which the payload can be applied for the real time application. The moving platform has been connected with the fixed platform by six linear actuators. Each of actuator has one cylinder $\mathrm{C}$ and one actuated piston. The cylinder has been attached with the fixed platform at the bottom point $B$ by spherical joint and end of the actuated piston has been connected with the top point $T$ by spherical joint at moving platform. Each actuator has been considered here as leg of this parallel manipulator.

The bottom points of actuators lie along the $O X Y$ horizontal plane. To describe the pose of platform, the stationary system has been taken as $O X Y Z$ where $Z$ is the upward vertical axis. The moving coordinate system opqr has been considered in such a way that the horizontal plane $o p q$ lies along the surface of mid-width of the $\mathrm{M}$. The initial vertical height between the fixed origin $O$ and moving origin $o$ has been taken as $h$. The initial pose of the platform has been called as the neutral pose where each of piston-extension has been considered as the mid of its maximum span of length.

The position vectors of bottom points and top points in the stationary coordinate $O X Y Z$ system have been defined as

$$
\begin{aligned}
\mathbf{x}_{B i} & =\left(\begin{array}{lll}
x_{B i} & y_{B i} & z_{B i}
\end{array}\right)^{T} \hat{\mathbf{e}}_{O}, \\
\text { and } \mathbf{x}_{T i} & =\left(\begin{array}{lll}
x_{T i} & y_{T i} & z_{T i}
\end{array}\right)^{T} \hat{\mathbf{e}}_{O},
\end{aligned}
$$

where unit vector $\hat{\mathbf{e}}_{O}$ corresponding to the $X, Y, Z$ directions

$$
\hat{\mathbf{e}}_{O}=\left(\begin{array}{lll}
\hat{i} & \hat{j} & \hat{k}
\end{array}\right)^{T} .
$$

The position vector of top points in the moving coordinate opqr system has been represented as

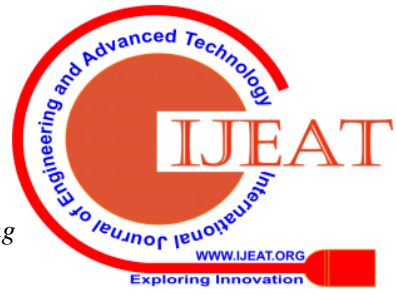




\section{Workspace Identification of Stewart Platform}

$$
\mathbf{p}_{T i}=\left(\begin{array}{lll}
p_{T i} & q_{T i} & r_{T i}
\end{array}\right) \hat{\mathbf{e}}_{o},
$$

with the unit vector $\hat{\mathbf{e}}_{o}$ corresponding to the $p, q$ and $r$ directions as

$$
\hat{\mathbf{e}}_{o}=\left(\hat{i}_{p} \hat{j}_{q} \hat{k}_{r}\right)^{T}
$$

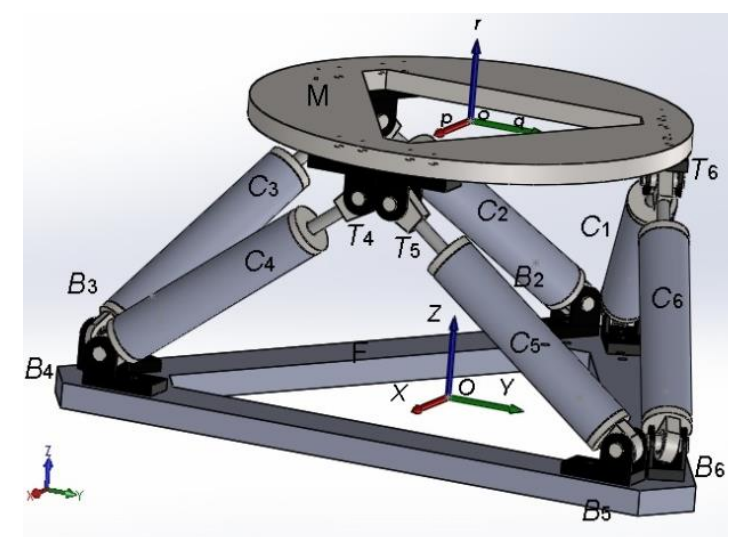

Fig. 1. Stewart Platform with fixed platform-F and moving platform-M connected with six linear cylinder-piston actuators.

The instantaneous pose of the platform is the combination of the translation motions surge, sway, heave along $X, Y, Z$ axis and rotational motions roll, pitch, yaw along $p, q, r$. The corresponding translation motion has been represented in the vector form as

$$
\mathbf{d}=\mathbf{x}_{o}^{T} \hat{\mathbf{e}}_{O}
$$

where $\mathbf{x}_{\mathbf{0}}$ is translation vector which is again defined as

$$
\mathbf{x}_{o}=\left(\begin{array}{lll}
x_{O} & y_{O} & z_{o}
\end{array}\right)^{T} .
$$

Similarly the rotational motions can be represented as

$$
\boldsymbol{\theta}=(\alpha \beta \gamma)^{T} \text {. }
$$

So the pose of the platform can be written with help of $(2 b)$ and $(2 \mathrm{c})$ as

$$
\mathbf{q}=\left(\begin{array}{ll}
\mathbf{d}^{T} & \boldsymbol{\theta}^{T}
\end{array}\right)^{T}=\left(\begin{array}{llllll}
x_{O} & y_{O} & z_{O} & \alpha & \beta & \gamma
\end{array}\right)^{T} .
$$

\section{INVERSE KINEMATIC MODELING FOR WORKSPACE IDENTIFICATION}

The mathematical formulation of the inverse kinematic modeling for Stewart Platform has been done in this section to find out the each leg motion for a corresponding motion of the moving platform. To find out each leg motion through inverse kinematic modeling, it is required to formulate the leg length vector which defines the distance between center of top joints and center of bottom joints with respect to the fixed coordinate.

The leg length vector has been defined with help of (1a) and (1b) as

$$
\mathbf{x}_{i}=\left(x_{i}=x_{T i}-x_{B i} \quad y_{i}=y_{T i}-y_{B i} z_{i}=z_{T i}-z_{B i}\right)^{T} \hat{\mathbf{e}}_{O} .
$$

So the distance between instantaneous center of top joint and the center of bottom joint has been defined in terms of leg length vector (3a) and unit vector (1c) as

$$
\mathbf{l}_{i}=\hat{\mathbf{e}}_{O}^{T} \mathbf{x}_{i} \text {. }
$$

Now the rotation by $\alpha$ about $p$ axis , $\beta$ about $q$ axis and $\gamma$ about $r$ axis have been incorporated in the leg length vector to make the rotational matrix $\mathbf{R}$ for transforming a vector from the moving to the stationary coordinate system [11]. This rotational matrix $\mathbf{R}$ has been defined as

$$
\mathbf{R}=\mathbf{R}_{\alpha} \mathbf{R}_{\beta} \mathbf{R}_{\gamma},
$$

where roll transformation $\mathbf{R}_{\alpha}$, pitch transformation $\mathbf{R}_{\beta}$ and yaw transformation $\mathbf{R}_{\gamma}$. These transformations have been adopted from Dasmahapatra et al. (2016) [12] as

$$
\begin{aligned}
& \mathbf{R}_{\alpha}=\left[\begin{array}{ccc}
1 & 0 & 0 \\
0 & \mathrm{c} \alpha & \mathrm{s} \alpha \\
0 & -\mathrm{s} \alpha & \mathrm{c} \alpha
\end{array}\right], \\
& \mathbf{R}_{\beta}=\left[\begin{array}{ccc}
\mathrm{c} \beta & 0 & -\mathrm{s} \beta \\
0 & 1 & 0 \\
\mathrm{~s} \beta & 0 & \mathrm{c} \beta
\end{array}\right], \\
& \text { and } \mathbf{R}_{\gamma}=\left[\begin{array}{ccc}
\mathrm{c} \gamma & \mathrm{s} \gamma & 0 \\
-\mathrm{s} \gamma & \mathrm{c} \gamma & 0 \\
0 & 0 & 1
\end{array}\right],
\end{aligned}
$$

where $\mathrm{c}$ and $\mathrm{s}$ represent cosine and sine function respectively. The transformations (3d-f) make rotational matrix (3c) as

$$
\mathbf{R}^{T}=\mathbf{R}_{\alpha}^{T} \mathbf{R}_{\beta}^{T} \mathbf{R}_{\gamma}^{T}=\left[\begin{array}{ccc}
\mathrm{c} \beta \mathrm{c} \gamma & -\mathrm{c} \beta \mathrm{s} \gamma & -\mathrm{s} \beta \\
\mathrm{c} \alpha \mathrm{s} \gamma+\mathrm{s} \alpha \mathrm{s} \beta \mathrm{c} \gamma & \mathrm{c} \alpha \mathrm{c} \gamma-\mathrm{s} \alpha \mathrm{s} \beta \mathrm{s} \gamma & -\mathrm{s} \alpha \mathrm{c} \beta \\
\mathrm{s} \alpha \mathrm{s} \gamma-\mathrm{c} \alpha \mathrm{s} \beta \mathrm{c} \gamma & \mathrm{s} \alpha \mathrm{c} \gamma+\mathrm{c} \alpha \mathrm{s} \beta \mathrm{s} \gamma & \mathrm{c} \alpha \mathrm{c} \beta
\end{array}\right] .
$$

Now using the equation (3b), the length of each leg can be formulated for the translation motion (2a-b) and rotational motion (2c) in terms of top points in moving coordinate (1d) and bottom points in the fixed coordinate (1a) as

$$
\mathbf{l}_{i}=\hat{\mathbf{e}}_{O}^{T}\left(\mathbf{R}^{T} \mathbf{p}_{T i}+\mathbf{d}-\mathbf{x}_{B i}\right)
$$

which leads to formulate the scalar form of each leg length as

$$
l_{i}=\sqrt{\left(\mathrm{p}_{T i}^{T} \mathrm{R}+\mathrm{d}^{T}-\mathrm{x}_{B i}^{T}\right)\left(\mathrm{R}^{T} \mathrm{p}_{T i}+\mathrm{d}-\mathrm{x}_{B i}\right)} .
$$

The above equation (3h) is the inverse kinematic equation from where the length of each leg could be calculated for a given translation and rotational motion by using the bottom and top points coordinates. This is the procedure how the piston length will be calculated from equation (3h). The corresponding actuator-piston motions for different platform pose have been depicted in the next section. 


\section{RESULTS AND DISCUSSION}

The workspace of the Stewart Platform has been depicted in this section with help of the inverse kinematic equations mentioned in the section III.

The workspace has been defined here as the different types of platform motions and the corresponding actuator-piston motions. The methodology which has been used here to find out the workspace identification from the inverse kinematic equations has been also described in this section.

\section{A. Methodology for workspace identification from inverse kinematic equations}

The maximum span of length of each actuator-piston of Stewart Platform has been taken from Dasmahapatra et al [12]. (2016) as $0.15 \mathrm{~m}$. The neutral pose of each motion has been taken as $0 \mathrm{~m}$ and the corresponding actuator piston position has been considered as $0.075 \mathrm{~m}$ which is mid of the maximum span of each actuator-piston. The workspace identifications in the Figs 2 to 7 have been performed in Matlab by using the inverse kinematic formulations mentioned in the Section III.

\section{B. Platform and Actuator-Piston Motions}

- Translation Motions- The individual actuator motion for three pure translation motions heaving, surging, swaying of sinusoidal type have been depicted in the Figs. 2 to 4 . The amplitude of each sinusoidal translation pose in the Figs. 2(b), 3(b) and 4(b) has been taken with $0.075 \mathrm{~m}$ and $0.5 \mathrm{~Hz}$ frequency. The corresponding piston motions of each translation pose have been depicted in the Figs. 2(a), 3(a) and 4(a). The Fig. 2(a) reveals that each of piston motions for heaving motion are identical. The amplitude of the stroking piston is about $0.04 \mathrm{~m}$ for heaving amplitude $0.075 \mathrm{~m}$. It can be exposed from the Fig. 2 that each piston motion starts from its initial position and initially extends then retracts. Though there are identical motions of each piston for heaving motion, but different motions of piston have been observed for other translation motions in Figs. 3 and 4 . During the upward surging, pistons 1 and 2 extend whereas pistons 3 and 6 retracts equally in the Fig. 3 . The lower stroking of pistons 4 and 5 for surging motion has been observed in the Fig. 3 (a). For the swaying motion in Fig. 4(b), the identical motion of pistons 1, 3 and 2, 6 have been observed in Fig. 4(a). The pistons 1, 3 and 4 are in-phase of swaying motion and other pistons are out of phase with swaying motion in the Fig. 4. The maximum and minimum displacements of actuator-pistons for translation motions in Figs. 2 to 4 have been tabulated in Table I.

Table- I: Maximum and minimum displacements of actuator-pistons for $\mathbf{0 . 0 7 5 m}$ sinusoidal translation motions

\begin{tabular}{|c|c|c|c|c|c|c|}
\hline \multirow{2}{*}{$\begin{array}{c}\text { Translation } \\
\text { Motion }\end{array}$} & \multicolumn{6}{|c|}{$\begin{array}{c}\text { Maximum and minimum displacements of } \\
\text { actuator-pistons in mm }\end{array}$} \\
\cline { 2 - 7 } & 1 & 2 & 3 & 4 & 5 & 6 \\
\hline \multirow{2}{*}{ Heaving } & 120, & 120, & 120, & 120, & 120, & 120, \\
& 30 & 30 & 30 & 30 & 30 & 30 \\
\hline Surging & 130, & 130, & 130, & 80, & 80, & 130, \\
& 25 & 25 & 25 & 75 & 75 & 25 \\
\hline Swaying & 110, & 110, & 110, & 135, & 135, & 110 \\
\hline
\end{tabular}

Retrieval Number: C5338029320/2020@BEIESP

\begin{tabular}{|l|l|l|l|l|l|l|}
\hline & 50 & 50 & 50 & 15 & 15 & 50 \\
\hline
\end{tabular}

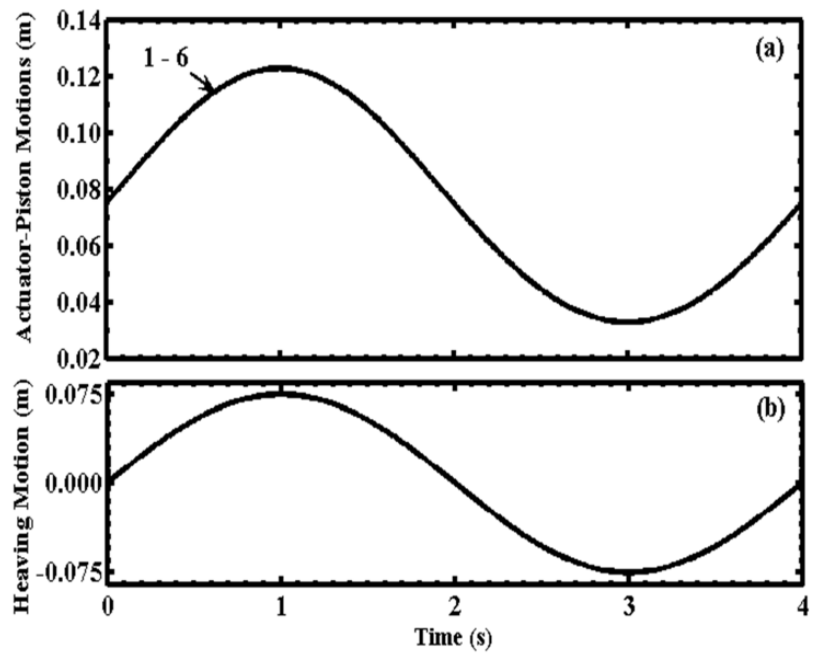

Fig. 2 Actuator motions for sinusoidal $0.075 \mathrm{~m}$ and $0.5 \mathrm{~Hz}$ Heaving motion of the Stewart Platform.
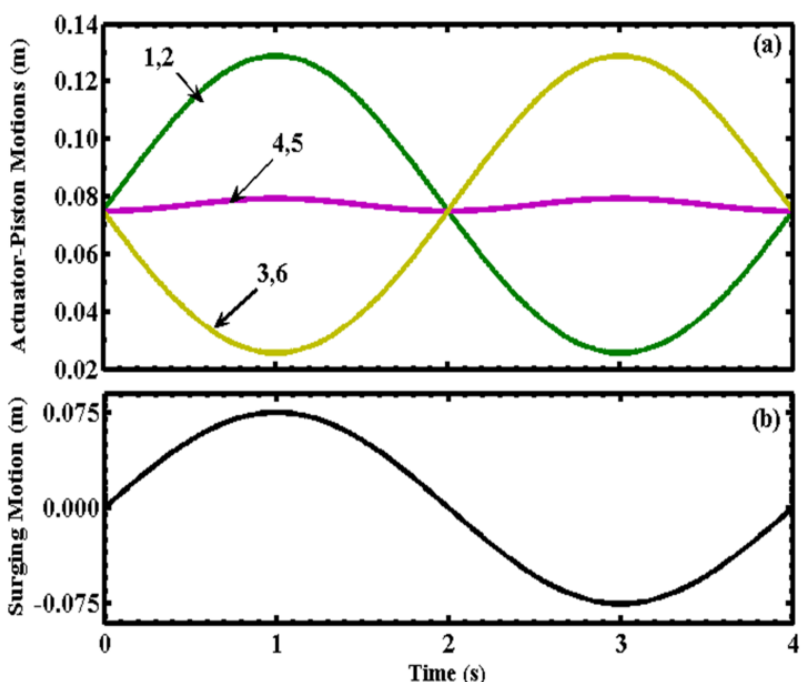

Fig. 3. Actuator motions for sinusoidal $0.075 \mathrm{~m}$ and $0.5 \mathrm{~Hz}$ Surging motion of the Stewart Platform.
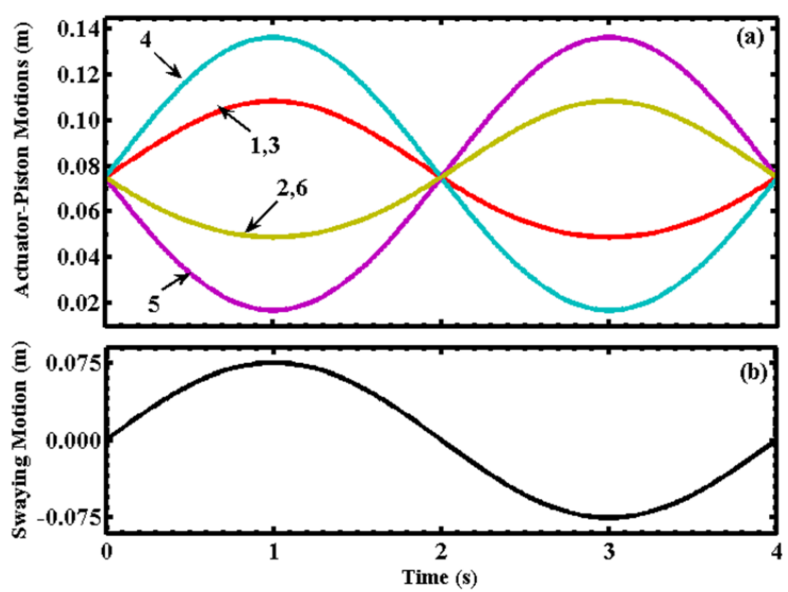

Fig. 4. Actuator motions for sinusoidal $0.075 \mathrm{~m}$ and $0.5 \mathrm{~Hz}$ Swaying motion of the Stewart Platform.

Published By:

Blue Eyes Intelligence Engineering \& Sciences Publication 
- Rotational Motions- The pure rotational motions rolling, pitching and yawing of sinusoidal type along with corresponding actuator motions have been represented in the Figs. 5 to 7. The sinusoidal rotational poses along with their corresponding actuator-piston motions have been portrayed in the Figs. 5 to 7. The amplitude of each rotational pose has been taken here as $10^{\circ}$ for the $0.5 \mathrm{~Hz}$ frequency. The motion of each piston in Fig. 5(a) is different for rolling motion depicted in Fig. 5(b). The pistons 1, 4, 6 are in-phase and 2, 3, 5 are out of phase with the rolling motion in Fig. 5. The maximum stroking length has been occurred for the pistons 1 and 2 but in different phase of each other. The pistons 4 and 5 show the minimum stroking tendency but in different phase of each other in Fig. 5(a). The pitching motion has been given in the Fig. 6(b) and the corresponding piston motions have been depicted in the Fig. 6(a). The pistons 1,2,3,6 are in-phase and the other pistons are out of phase with pitching motion in Fig. 6. The maximum stroking pistons are 4, 5 and minimum stroking pistons 1,2 for pitching motion. The stroking length of each piston are identical for yawing motion in Fig. 7 but the pistons 2, 4, 6 are in-phase and others are out of phase of the yawing motion. The maximum and minimum displacements of actuator-pistons for rotational motions in Figs. 5 to 7 have been tabulated in the Table II.

Table- II: Maximum and minimum displacements of actuator-pistons for $10^{0}$ sinusoidal rotational motions

\begin{tabular}{|c|c|c|c|c|c|c|}
\hline \multirow{2}{*}{$\begin{array}{c}\text { Translation } \\
\text { Motion }\end{array}$} & \multicolumn{6}{|c|}{$\begin{array}{c}\text { Maximum and minimum displacements of } \\
\text { actuator-pistons in mm }\end{array}$} \\
\cline { 2 - 7 } & 1 & 2 & 3 & 4 & 5 & 6 \\
\hline \multirow{2}{*}{ Rolling } & 115, & 115, & 110, & 85, & 85, & 110, \\
& 40 & 40 & 50 & 70 & 70 & 50 \\
\hline \multirow{2}{*}{ Pitching } & 90, & 90, & 100, & 120, & 120, & 100, \\
& 70 & 70 & 50 & 40 & 40 & 50 \\
\hline \multirow{2}{*}{ Yawing } & 125, & 125, & 125, & 125, & 125, & 125, \\
& 25 & 25 & 25 & 25 & 25 & 25 \\
\hline
\end{tabular}
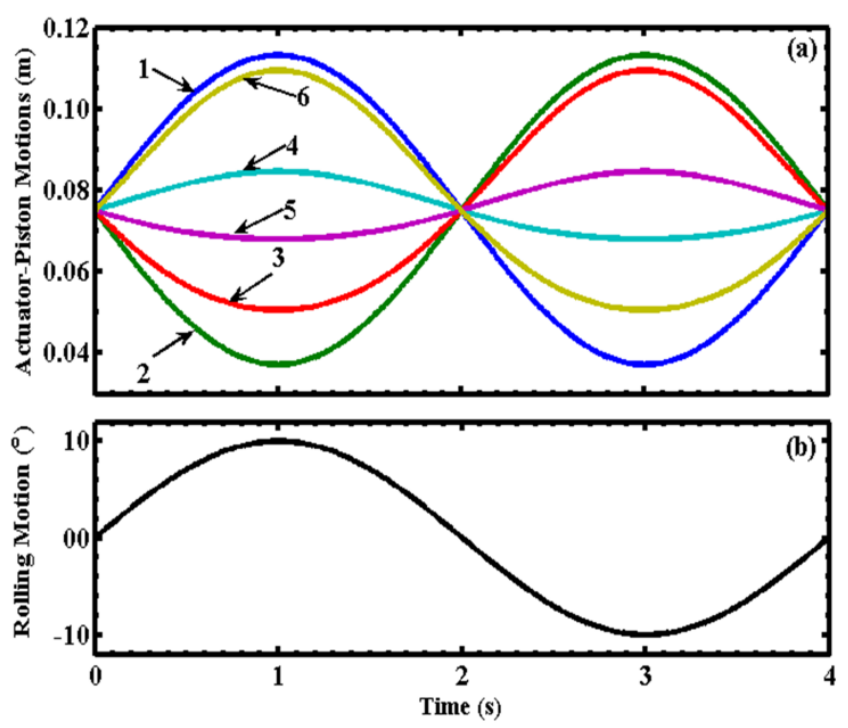

Fig. 5. Actuator motions for sinusoidal $10^{\circ}$ and $0.5 \mathrm{~Hz}$ Rolling motion of the Stewart Platform.
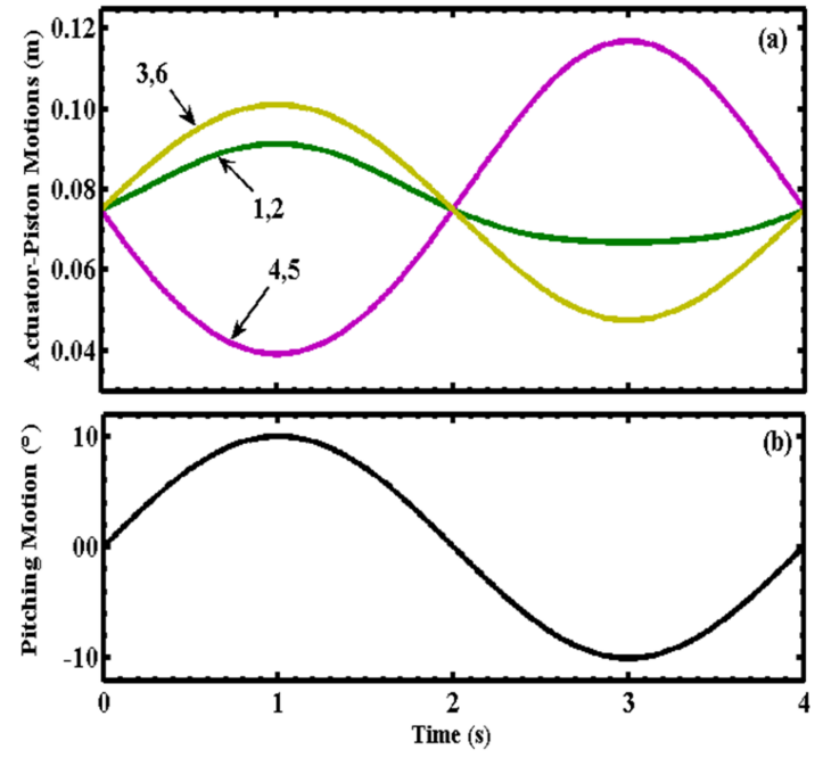

Fig. 6. Actuator motions for sinusoidal $10^{\circ}$ and $0.5 \mathrm{~Hz}$ Pitching motion of the Stewart Platform.

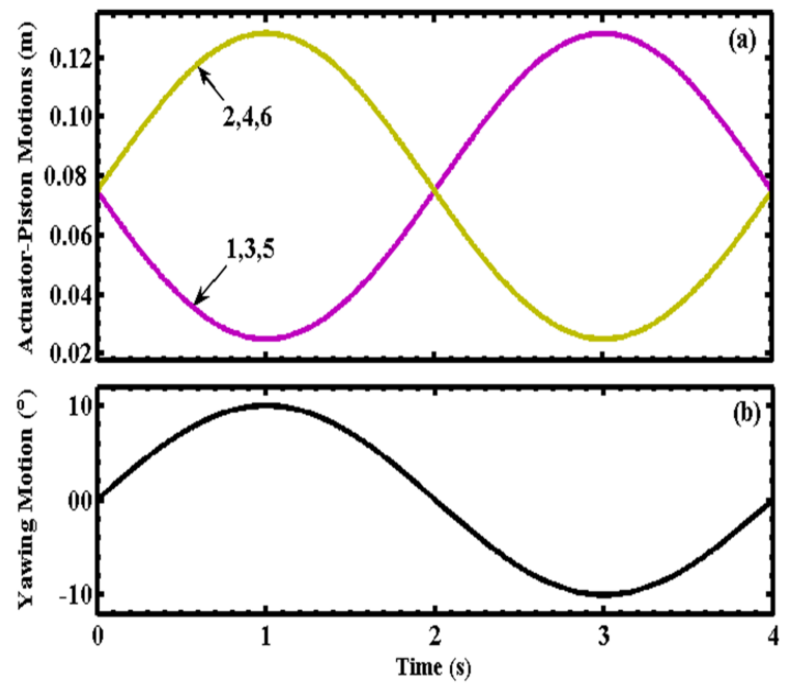

Fig. 7. Actuator motions for sinusoidal $10^{\circ}$ and $0.5 \mathrm{~Hz}$ Yawing motion of the Stewart Platform.

\section{CONCLUSION}

The mathematical modeling of the inverse kinematic for the 6-DOF Stewart Platform has been successfully implemented for finding out the workspace of parallel manipulator Stewart Platform. The motions of the actuator-pistons for the corresponding translation and rotational poses have been studied and their corresponding motion-tendency have been identified. The range of each piston for translation and rotational motions has been tabulated in this work. The real time experiments for the corresponding platform poses can be performed in future.

\section{ACKNOWLEDGMENT}

We sincerely acknowledge the supports of Mechanical Engineering Department of Jadavpur University to inspire and formulate kinematic modelling of the Stewart Platform.

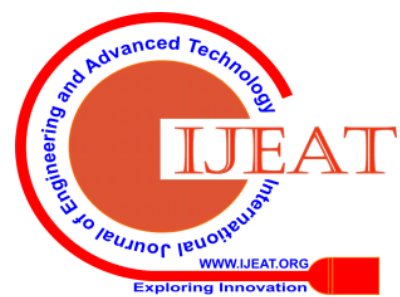




\section{REFERENCES}

1. S. Tafazoli, CW. de Silva and PD. Lawrence, "Tracking control of an electro hydraulic manipulator in the presence of friction,” IEEE Trans. on Control System Technology, vol. 6, 1998, pp. 401-411.

2. M. Qiang, Z. Tao, He. Jing-feng, S. Jing-yan and H. Jun-wei, "Dynamic modeling of a 6-degree-of-freedom Stewart platform driven by a permanent magnet synchronous motor," Journal of Zhejiang University-SCIENCE C (Computers \& Electronics), vol. 11, 2010, pp. 751-761.

3. J-P. Merlet, Parallel Robots, Kluwer Academic Publisher, Netherlands, 2000.

4. N-I. Kim and C-W. Lee, "Multi-axis vibration control of a slender structure by a Stewart platform manipulator," Mechanisms and Machines Theory, Vol. 36, 2001, pp. 1253-1269.

5. YX. Su and BY . Duan, "The application of the Stewart platform in large spherical radio telescopes," J Robotic Systems, vol. 17, 2000, pp. 375-83.

6. YX. Su, BY. Duan, B. Peng, RD. Nan, "Mechatronics design of response enhancement of Stewart fine tuning platform for the square kilometer array," Mechatronics, vol. 13, 2003, pp. 111-125.

7. A. Preumont, M. Horodinca, I. Romanescu, B . Marneffe, M. Avraam, A. Deraemaeker, F. Bossens, AA. Hanieh, "A six-axis single-stage active vibration isolator based on Stewart platform," Journal of Sound and Vibration, vol. 300, 2007, pp. 644-661.

8. D. Stewart, "A platform with six degrees of freedom. Proceedings of Institute of Mechanical Engineering," Vol. 180, 1965, pp. 371-386.

9. L. Fraguela, L. Fridman, V.V. Alexandrov, "Output integral sliding mode control to stabilize position of a Stewart platform," Journal of the Franklin Institute, Vol. 349, 2012, pp. 1526-1542.

10. W. Matthias, U. Volker, W. Thomas, S. Jan, D. Mark, and H. Andrew, "A Stewart Platform for Precision Surgery," Transaction of the Institute of Measurement and Control, vol. 25, 2003, pp. 329-334.

11. MD. Shuster, Spacecraft attitude determination and control, Fundamentals of Space Systems: Oxford University Press, 1994, pp. 245-336.

12. S. Dasmahapatra, D. Saha, R. Saha, D. Sanyal, D. Lahiri, and J.P. Sing, "Analysis of 6-DOF motion with PI controller in electrohydraulic Stewart platform," 2016 IEEE First International Conference on Control, Measurement and Instrumentation (CMI). DOI: 10.1109/CMI.2016.7413717, 2016, pp. 186-190.

\section{AUTHORS PROFILE}

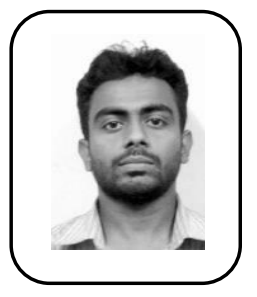

Dr. Sibsankar Dasmahapatra, Received the Bachelor's Degree in Mechanical Engineering from Kalyani Govt Engineering College, India in 2007. He has obtained Masters and PhD in Mechanical Engineering from Jadavpur University. In 2016, he has joined as Assistant Professor in Mechanical Engineering Department, Kalyani Govt Engineering College. His research interest on electro-hydraulic actuation system and its control.

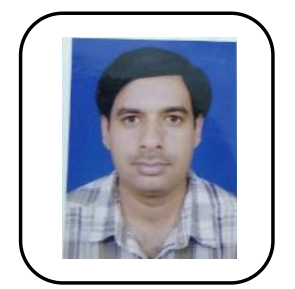

Mr. Mintu Ghosh, Received the Bachelor's degree in Mechanical Engineering from Visvesvaraya Technological University(VTU), Karnataka, India, in 2003 and Master's degree in Design and Production from NIT Durgapur, India in 2006. He joined the Mechanical Engineering Department, Bengal College of Engineering and Technology, Durgapur in 2007 and presently research work on Electro-hydraulics. working as Assistant Professor. He is pursuing 